\title{
Contrast-enhanced ultrasonography follow-up after radiofrequency ablation in normal rabbit kidney
}

\author{
Rong $\mathrm{Wu}^{1}$, Fan-hua $\mathrm{Xu}^{2}$, Ming-hua $\mathrm{Yao}^{1}$, Juan $\mathrm{Xie}^{1}$, Bing $\mathrm{Hu}^{3}$ \\ 1Department of Ultrasound in Medicine, Shanghai tenth People's Hospital, \\ Tongji University School of Medicine, Shanghai, China \\ 2Department of Ultrasound in Medicine, the Second Clinical Medical College of Jinan \\ University, Shenzhen People's Hospital, Shenzhen, China \\ 3Department of Ultrasound in Medicine, Shanghai Jiao Tong University affiliated Sixth \\ People's Hospital, Shanghai, China \\ Submitted: 13 April 2012 \\ Accepted: 21 November 2012 \\ Arch Med Sci 2013; 9, 4: 608-613 \\ DOI: 10.5114/aoms.2013.37271 \\ Copyright $\odot 2013$ Termedia \& Banach \\ Corresponding author: \\ Bing $\mathrm{Hu}$ \\ Department \\ of Ultrasound in Medicine \\ Shanghai Jiao Tong University \\ affiliated Sixth \\ People's Hospital \\ Shanghai 200233, China \\ No. 600 , Yishan Road \\ Shanghai 200233, China \\ Phone: 0086-13817031977 \\ E-mail: binghu1959@ \\ hotmail.com
}

\begin{abstract}
Introduction: The aim of this study was to evaluate the diagnostic performance of SonoVue-enhanced ultrasonography in the follow-up of rabbit kidney lesions induced by percutaneous radiofrequency ablation.

Material and methods: New Zealand rabbits (28) underwent percutaneous radiofrequency ablation (RFA) to generate renal lesions. Lesions were evaluated by conventional 2D ultrasound and contrast-enhanced ultrasonography (CEUS) at 1 day, 1 week, 1 month and 3 months after RFA, and the results were compared to gross pathology.

Results: One day and 1 week after RFA, renal lesions were wedge-shaped in the gross dissection cross-sectional profiles. Conventional ultrasound could not indicate the extent of the lesions; however, CEUS could exactly delineate the lesion shape and size. At 1 and 3 months, lesions were observed as hyperechogenic areas on conventional ultrasound, and as small perfusion defects on CEUS. The differences in the lesion measurements obtained by CEUS and in pathological specimens were not statistically significant $(p>0.05)$.

Conclusions: The conclusion could be deduced from the study that SonoVueenhanced ultrasonography was effective for the follow-up of normal rabbit kidney percutaneous radiofrequency ablation.
\end{abstract}

Key words: contrast-enhanced ultrasonography, radiofrequency ablation, kidney, intervention.

\section{Introduction}

During the past decade, kidney disease was seen among a large amount of the world's population, which was an enormous burden on scarce health care resources and health insurance payments [1, 2]. In the United States, 58,240 new cases of renal cancer were diagnosed in 2010, with 13,040 deaths, accounting for $3 \%$ of both new cancer diagnoses and deaths that year [3]. The widespread use of various imaging modalities has led to an increase in the number of renal masses incidentally detected $[4,5]$.

In recent years, our therapeutic options have changed from radical nephrectomy to partial nephrectomy for neoplasms smaller than $4 \mathrm{~cm}$. With the progress of the ablation technology, more minimally invasive technology has developed such as radiofrequency ablation [6, 7], highintensity focused ultrasound [8] and microwave $[9,10]$. Percutaneous 
radiofrequency ablation (RFA) of renal tumors is a minimally invasive technique for the treatment of patients unsuitable for surgery due to serious comorbidities $[11,12]$. Although long-term follow-up has not been reported, previous studies have indicated the safety and feasibility of RFA for treatment of renal small cell carcinoma in humans [13-15].

As RFA is an in situ non-surgical method of tumor destruction, in which surgical margins cannot be determined, imaging is important for the assessment of RFA results. Imaging modalities such as ultrasound, contrast-enhanced computed tomography $(\mathrm{CT})$ and magnetic resonance imaging (MRI) have been widely used to assess treatment efficacy after RFA $[16,17]$. With the development of contrast-specific imaging techniques and increasing availability of contrast agents, the novel imaging modality contrast-enhanced ultrasonography (CEUS) has been an important recent development in clinical ultrasound [18-20]. Contrast-enhanced ultrasonography is widely used for the diagnosis of local lesions and evaluation of treatment effectiveness, especially in liver tumors [21, 22].

The objective of the present study was to compare the diagnostic performance of CEUS to histopathological analysis, after percutaneous RFA in normal rabbit kidney.

\section{Material and methods}

\section{Animals}

The protocol was approved by the Institutional Animal Use and Care Committee. Experiments were performed with 28 healthy male or female New Zealand white rabbits weighing between $2.8 \mathrm{~kg}$ and $3.5 \mathrm{~kg}$ (Experimental Animal Center, Shanghai, China). Animals were allowed to acclimatize for at least 5 days prior to the experiment and were kept in a closed system with environmental temperature adjusted to $18-20^{\circ} \mathrm{C}$, and a non-regulated relative air humidity of approximately $60 \%$. Animals were provided food and water ad libitum during the entire study. The 28 rabbits were randomly divided into four groups depending on the duration of analysis after RFA, as follows: 1 day, 1 week, 1 month and 3 months after RFA.

\section{Surgical procedure}

Animals were anesthetized by ear vein injection with $30 \mathrm{mg} / \mathrm{kg}$ 5\% pentobarbital sodium solution (Shanghai Pharmacy, Shanghai, China). The hair in the bilateral renal region was removed by shaving the skin with clippers. A 24-gauge angiocatheter was used to establish an intravenous line in the ear vein. An electrode plate was pasted on the right side of the abdomen and connected to the radio frequency ablation device. Rabbits were placed in a right lateral decubitus position and RFA was administered with a Model 1500 generator, using the StarBurst SDE probe (RITA Medical Systems, Mountain View, CA, USA) to create a lesion. Briefly, a radiofrequency electrode needle was percutaneously inserted into the left kidney under ultrasonography guidance, with the needle parallel to the probe during the insertion. RF energy was delivered for $5 \mathrm{~min}$ at a target temperature between $75^{\circ} \mathrm{C}$ and $85^{\circ} \mathrm{C}$. After RFA, conventional power Doppler and CEUS were performed on the kidney lesion. The diameter of the thermal lesions was measured using CEUS after each RFA. Animals were returned to the cage after the operation.

\section{Contrast-enhanced ultrasonography}

Ultrasonography and contrast-enhanced ultrasonography (CEUS) were performed 1 day, 1 week, 1 month and 3 months after RFA using the MPX ultrasound system (Esaote S.p.A., Genoa, Italy) with an LA523 probe and another LA523E contrast transducer at a frequency of $12.5 \mathrm{MHz}$. A 41-mm depth was set and other parameters, such as time gain compensation (TGC) and focus range, were debugged to their optimal parameters according to the actual situation. The optimal lesion scanning positions were selected before switching to CEUS mode. SonoVue (Bracco, Italy) was used as the contrast agent, $\mathrm{MI}=0.106$. The contrast agent was diluted with saline solution to $5 \mathrm{ml}$ and infused through the rabbit ear vein in boluses of $0.1 \mathrm{ml} / \mathrm{kg}$ followed immediately by $2 \mathrm{ml}$ of physiological saline. Dynamic imaging data were saved to disk and three physicians experienced in CEUS reviewed the imaging data after the procedure and measured the maximum diameter parallel to the renal axis.

\section{Histopathological analysis}

All histopathological analyses were performed by a pathologist blinded to the RFA lesion sonography measurements. Seven rabbits were euthanized at 1 day, 1 week, 1 month and 3 months after RFA, and the kidneys were removed and sequentially sectioned in the coronal plane at a thickness of approximately $4 \mathrm{~mm}$. The boundary between the ablated lesions and normal tissue was distinguished by staining the tissue slices in $1 \%$ triphenyl tetrazolium chloride, a redox indicator which is enzymatically reduced in viable tissue, for $5 \mathrm{~min}$ to $10 \mathrm{~min}$ to distinguish between viable and nonviable cells and definitely visualize the thermal coagulation. The maximum diameter of coagulative necrosis parallel to the renal axis was measured with a ruler.

\section{Statistical analysis}

All data were expressed in millimeters as mean \pm standard deviation. The Wilcoxon matched pair rank test was used to evaluate the differences in 
the conventional ultrasound, CEUS and pathological specimen measurements of the ablated lesions using SPSS 11.0 (IBM, Armonk, NY, USA).

\section{Results}

One day and 1 week after RFA, thermal lesions showed only slight hyperechogenicity or no obvious changes on conventional gray-scale ultrasonography, and the thermal lesion margins were poorly differentiated (Figure $1 \mathrm{~A}$ ). Color and power Doppler indicated a deficiency in blood flow through the ablated lesions, via which the exact location of the ablated lesions could be accurately determined; however, this area tended to overestimate the ablated region. On CEUS, the ablated lesions appeared as wedge-shaped hypoechoic regions pointing to the renal hilum (Figure $1 \mathrm{~B}$ ). The differences in the absolute lesion measurements determined by CEUS and in the pathological specimens at 1 day and 1 week after RFA were not statistically significant $(p>0.05$, Table I).

On gross dissection at 1 week, the RFA lesions were observed as cone-shaped areas, with wedge-
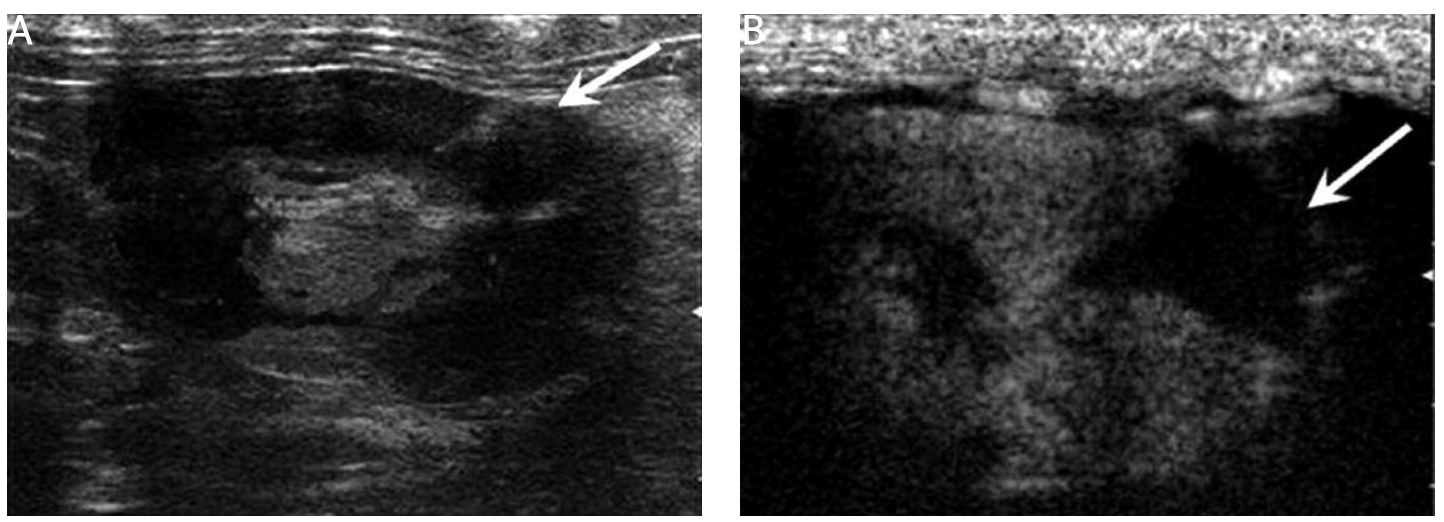

shaped cross-sectional profiles (Figure $1 \mathrm{C}$ ). One day after RFA ablation, the needle tract could be observed as gray tissue at the center of the lesion (data not shown), surrounded by an acroteric red hyperemia peripheral area with a thickness of 2.03 $\pm 0.50 \mathrm{~mm}$. One week after RFA, the color of the ablated lesions deepened and the needle tract could still be observed at the center of the lesion, while the acroteric red hyperemia peripheral range narrowed to a thickness of $0.66 \pm 0.15 \mathrm{~mm}$.

One month and 3 months after RFA, the lesions showed as hyperechoic nodules with a round or oval shape on conventional ultrasound (Figure $2 \mathrm{~A}$ ). Some lesions had an obvious posterior acoustic shadow with a clear border, which was closely linked to the surrounding perirenal fat and focal renal capsule shrinkage. Color and power Doppler indicated that renal perfusion was good and no significant defects in blood flow could be observed. Contrast-enhanced ultrasonography indicated small focal perfusion defects at the lesion sites, while the rest of the kidney was well perfused (Figure 2 B). On gross dissection, the hyperechoic structure was observed as a hard fibrous callus with adhesions of the ablated

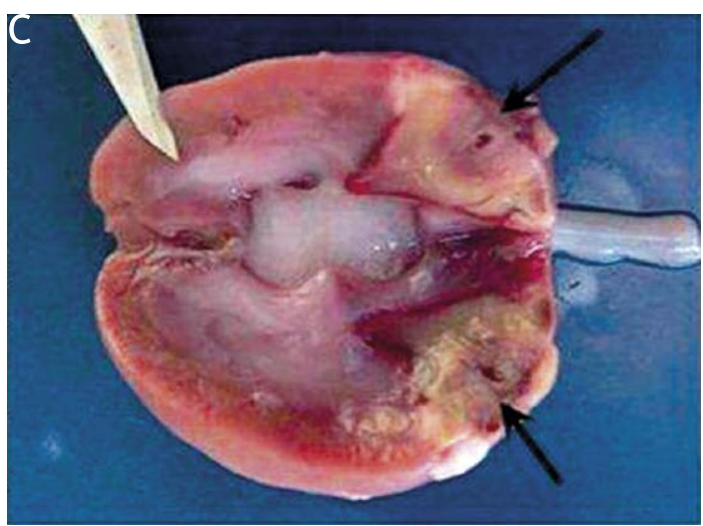

Figure 1. Image of the same rabbit kidney 1 week after RFA: A - by conventional grayscale ultrasonography, therma lesion margin (white arrow) is poorly demarcated; B - contrast-enhanced ultrasonography showing clear demarcation of thermal lesion borders; lesion appears as hypoechoic area (white arrow) surrounded by contrast-enhanced hyperechoic kidney parenchyma; $\mathrm{C}$ - gross dissection, the lesions are cone-shaped in the cross-section. The coagulated tissues (black arrow) correspond to the hypoechoic area in B 
Table I. Comparison of mean \pm standard deviation radiofrequency lesion measurements using conventional ultrasound, CEUS and gross pathology with triphenyl tetrazolium chloride staining

\begin{tabular}{|lccc|}
\hline $\begin{array}{l}\text { Time } \\
\text { after } \\
\text { RFA }\end{array}$ & $\begin{array}{c}\text { Conventional } \\
\text { ultrasound } \\
\text { [mm] }\end{array}$ & $\begin{array}{c}\text { CEUS } \\
{[\mathrm{mm}]}\end{array}$ & $\begin{array}{c}\text { Gross } \\
\text { pathology } \\
\text { [mm] }\end{array}$ \\
\hline 1 day & $\mathrm{ND}$ & $13.8 \pm 3.21^{\star}$ & $13.7 \pm 2.61$ \\
\hline 1 week & $\mathrm{ND}$ & $16.5 \pm 5.45^{\star}$ & $15.7 \pm 4.89$ \\
\hline 1 month & $8.89 \pm 2.79^{\star \star}$ & $5.81 \pm 1.47^{\star}$ & $5.84 \pm 1.51$ \\
\hline 3 months & $9.37 \pm 2.13^{\star \star}$ & $3.41 \pm 0.79^{\star}$ & $3.43 \pm 0.56$ \\
\hline
\end{tabular}

Note: Wilcoxon matched pair rank test: ${ }^{*} p>0.05,{ }^{* *} p<0.05$, compared to gross pathology. ND - could not be determined using conventional ultrasound, CEUS - contrast-enhanced ultrasonography

lesions to perirenal fat (Figure $2 \mathrm{C}$ ). The extent of focal hyperechoic echo indicated by conventional ultrasound was larger than the renal fiber nodule measurements in pathological specimens $(p<0.05)$. The differences in absolute lesion measurements obtained by CEUS and in pathological specimens 1 month and 3 months after RFA were not statistically significant $(p>0.05$, Table I).
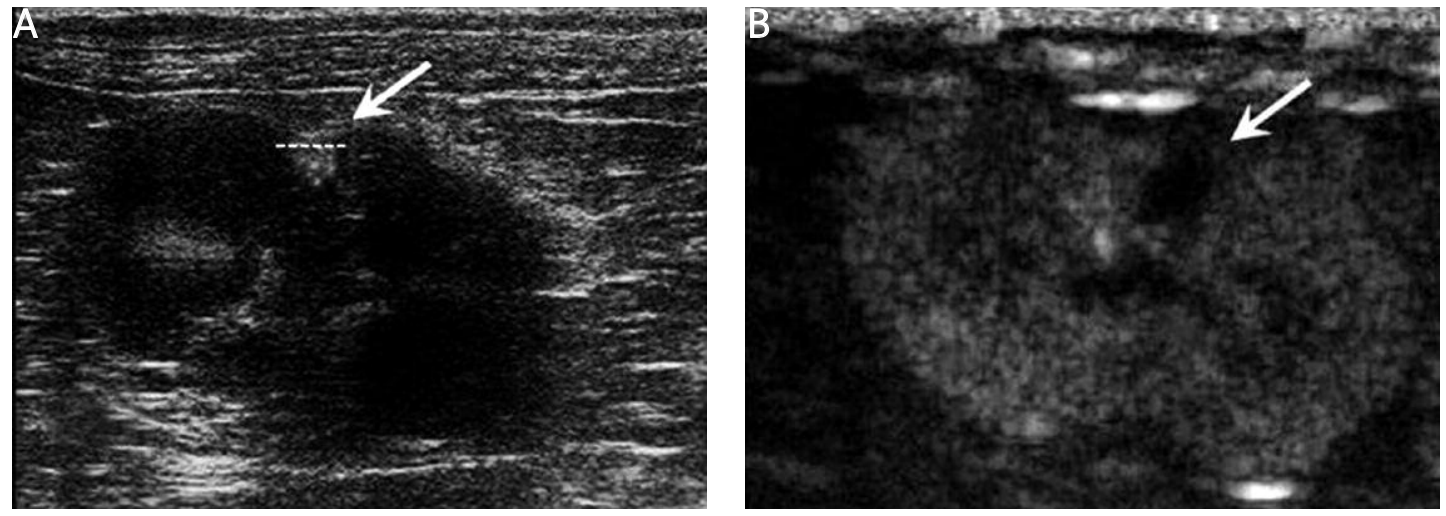

\section{Discussion}

Ultrasound, CT and MRI are usually used for follow-up after RFA in renal tumor treatment. Some investigators have reported that the volume and size of lesions determined by MRI and pathological examination correlate well; however, ultrasound has some advantages over MRI. It is available at the point of care and the purchase of equipment is a small fraction of the cost of MRI, typically $10 \%$. It also has fewer restrictions with regards to its use for patients with implants or those who are very unwell. Gervais et al. did not observe a correlation in the results of ultrasound, CT or enhanced MRI imaging analyses of renal and liver tumors, which manifest in a similar manner after RFA [23].

Radiofrequency (RF) releases high-frequency alternating current from a non-insulated electrode tip into the surrounding tissues, giving rise to ionic vibration and frictional heating in the tissue around the electrode [24]. The tissue undergoes coagulative necrosis, capillaries embolize in the necrotic area and the focal microcirculation is disturbed. With the development of contrast-specific imaging techniques and the availability of contrast

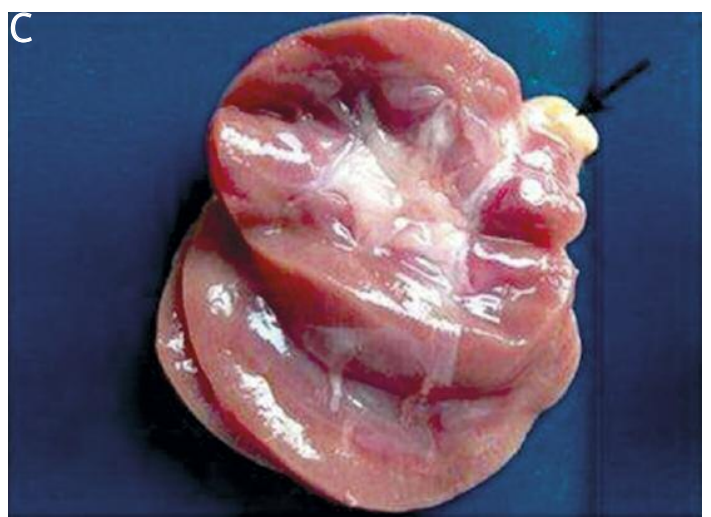

Figure 2. Image of the same rabbit kidney 3 months after RFA: A - by conventional grayscale ultrasonography, the arrow shows oval-shaped hyperechoic nodules; $\mathbf{B}$ - contrast-enhanced ultrasonography showing clear demarcation of thermal lesion borders; with the lesion appearing as a hypoechoic area (white arrow) surrounded by contrast-enhanced hyperechoic renal parenchyma; $\mathrm{C}$ - gross dissection showed that in hard fibrous callus lesions are visible on cross-sectioning, formed by adhesions of adjacent renal-ablated lesions to perirenal fat. The coagulated tissues (black arrow) correspond to the hypoechoic area in $\mathrm{B}$ 
agents, CEUS has been assessed in many indications. Microbubble contrast agents are vascular pool agents with a small diameter that enables them to pass into the capillary bed [18]. On CEUS, the ablated lesions showed as hypoechoic regions because of the microbubble contrast agent perfusion defects at the lesion site. Thus, the accurate evaluation of the microcirculation in the ablated area is feasible using CEUS. Moreover, CEUS can be used in patients with iodine allergy and impaired renal function, which may contraindicate contrast-enhanced CT or MRI [25].

This study demonstrates that, by an evaluation of the focal microcirculation, CEUS can be used to assess the extent of radiofrequency ablated lesions. One day and 1 week after RFA, conventional ultrasonography could not locate ablated lesions and color and power Doppler could not accurately demonstrate the extent of ablated lesions. Other researchers have reported that conventional ultrasonography cannot clearly demonstrate the boundary between thermal lesions and surrounding tissue $[26,27]$. Color or power Doppler ultrasound have limited ability to detect capillary and low speed flow, and Rendon et al. demonstrated that gray-scale and power Doppler ultrasound were not helpful in the detection or measurement of ablated lesions [28]. We found that CEUS could indicate the contours of the ablated lesions via defects in microcirculatory perfusion. Lesion measurements taken from CEUS were consistent with gross and histological measurements, which indicated that real-time monitoring of RFA treatment in renal cancer using CEUS is feasible. The ablated lesions appeared wedge-shaped in the cross-sectional dissection, probably due to the distribution and damage of segmental renal arteries, as artery branch occlusion after RFA will lead to renal infarct in the areas supplied by occluded vessels. This indicates that the damage to normal renal parenchyma after RFA was the result of both thermal injury and segmental blood flow occlusion.

By 1 month and 3 months after RFA, the number of ablated lesions had gradually decreased, neither fibrosis nor calcification was observed, and both the area of focal hyperechogenicity and the renal capsule had decreased in size. Hard calluses, formed by adhesions of areas adjacent to the ablated renal contour to perirenal fat, were observed on gross dissection. The measurements of hyperechoic structure on conventional ultrasound were significantly larger than the measurements obtained by gross pathology, which may have been due to measurement of adhesions; this could not be differentiated on conventional ultrasound. At 1 month and 3 months, CEUS indicated perfusion defects at the lesion site, while the remainder of the kidney area had good blood perfusion. Conventional ultrasound could approximately indicate the extent of fibrosis, while CEUS more accurately showed the size of fibrous nodules via the areas of perfusion defects. This indicated that CEUS can act in complement and as an extension to conventional ultrasound.

In conclusion, our study suggests that CEUS can accurately assess the extent of coagulative necrosis and is effective in the follow-up of normal rabbit kidney after percutaneous RFA. Contrast-enhanced ultrasonography could accurately evaluate the effect of RFA, which is consistent with previous reports [29]. Compared with pathological lesions, we observed differentiation of the residual tumor and the ablation area of rabbit implanted tumor after RFA using CEUS. We will continue these comparative imaging studies in human renal tumors after RFA therapy.

\section{Acknowledgments}

Rong Wu and Fan-hua Xu contribute equally to this work.

This work was supported by the Department of Pathology, Shanghai Jiao Tong University affiliated Sixth People's Hospital, Shanghai 200233, China.

\section{References}

1. Couser WG, Riella MC. World Kidney Day 2011 - Protect your kidneys, save your heart. Arch Med Sci 2011; 7: 1-4.

2. Machnicki G, Lentine KL, Salvalaggio PR, Burroughs TE, Brennan DC, Schnitzler MA. Kidney transplant Medicare payments and length of stay: associations with comorbidities and organ quality. Arch Med Sci 2011; 7: 278-86.

3. Jemal A, Siegel R, Xu J, Ward E. Cancer statistics, 2010. CA Cancer J Clin 2010; 60: 277-300.

4. Jayson M, Sanders $H$. Increased incidence of serendipitously discovered renal cell carcinoma. Urology 1998 51: 203-5.

5. Luciani LG, Cestari R, Tallarigo C. Incidental renal cell carcinoma-age and stage characterization and clinical implications: study of 1092 patients (1982-1997). Urology 2000; 56: 58-62.

6. Zlotta AR, Wildschutz T, Raviv G, et al. Radiofrequency interstitial tumor ablation (RITA) is a possible new modality for treatment of renal cancer: ex vivo and in vivo experience. J Endourol 1997; 11: 251-8.

7. Zagoria RJ, Traver MA, Werle DM, Perini M, Hayasaka S, Clark PE. Oncologic efficacy of CT-guided percutaneous radiofrequency ablation of renal cell carcinomas. AJR Am J Roentgenol 2007; 189: 429-36.

8. Ritchie RW, Leslie T, Phillips R, et al. Extracorporeal high intensity focused ultrasound for renal tumours: a 3-year follow-up. BJU Int 2010; 106: 1004-9.

9. Carrafiello G, Mangini M, Fontana F, et al. Single-antenna microwave ablation under contrast-enhanced ultrasound guidance for treatment of small renal cell carcinoma: preliminary experience. Cardiovasc Intervent Radiol 2010; 33: 367-74.

10. Liang P, Wang Y, Zhang D, Yu X, Gao Y, Ni X. Ultrasound guided percutaneous microwave ablation for small renal cancer: initial experience. J Urol 2008; 180: 844-8.

11. McAchran SE, Lesani OA, Resnick MI. Radiofrequency ablation of renal tumors: past, present, and future. Urology 2005; 66: 15-22. 
12. Gervais DA, McGovern FJ, Arellano RS, McDougal WS, Mueller PR. Renal cell carcinoma: clinical experience and technical success with radio-frequency ablation of 42 tumors. Radiology 2003; 226: 417-24.

13. Zlotta AR, Djavan B, Matos C, Noel JC, Peny MO, Silverman DE. Percutaneous transperineal radiofrequency ablation of prostate tumour: safety, feasibility and pathological effects on human prostate cancer. Br J Urol 1998; 81: 265-75.

14. Carraway WA, Raman JD, Cadeddu JA. Current status of renal radiofrequency ablation. Curr Opin Urol 2009; 19: 143-7.

15. Mues AC, Landman J. Image-guided percutaneous ablation of renal tumors: outcomes, technique, and application in urologic practice. Curr Urol Rep 2010; 11: 8-14.

16. Rutherford EE, Cast JE, Breen DJ. Immediate and longterm $\mathrm{CT}$ appearances following radiofrequency ablation of renal tumors. Clin Radiol 2008; 63: 220-30.

17. Zagoria RJ, Traver MA, Werle DM, Perini M, Hayasaka S, Clark PE. Oncologic efficacy of CT-guided percutaneous radiofrequency ablation of renal cell carcinomas. Am J Roentgenol 2007; 189: 429-36.

18. Wink MH, Wijkstra H, De La Rosette JJ, Grimbergen CA. Ultrasound imaging and contrast agents: a safe alternative to MRI? Minim Invasive Ther Allied Technol 2006; 15: 93-100.

19. Xu ZF, Xu HX, Xie XY, Liu GJ, Zheng YL, Lu MD. Renal cell carcinoma and renal angiomyolipoma: differential diagnosis with real-time contrast-enhanced ultrasonography. J Ultrasound Med 2010; 29: 709-17.

20. Xu ZF, Xu HX, Xie XY, et al. Renal cell carcinoma: real-time contrast-enhanced ultrasound findings. Abdom Imaging 2010; 35: 750-6.

21. Salvaggio G, Campisi A, Lo Greco V, et al. Evaluation of post treatment response of hepatocellular carcinoma: comparison of ultrasonography with second-generation ultrasound contrast agent and multidetector CT. Abdom Imaging 2010; 35: 447-53.

22. Bartolotta TV, Taibbi A, Midiri M, De Maria M. Hepatocellular cancer response to radiofrequency tumor ablation: contrast-enhanced ultrasound. Abdom Imaging 2008; 33: 501-11.

23. Gervais DA, McGovern FJ, Wood BJ, Goldberg SN, McDougal WS, Mueller PR. Radio-frequency ablation of renal cell carcinoma: early clinical experience. Radiology 2000; 217: 665-72.

24. Goldberg SN, Gazelle GS, Mueller PR. Thermal ablation therapy for focal malignancy: a unified approach to underlying principles, techniques, and diagnostic imaging guidance. Am J Roentgenol 2000; 174: 323-31.

25. Yang B, Fu NH, Shen DJ, Liu P, Meng QX. Contrastenhanced ultrasound in the characteristics of renal tumors [Chinease]. Chin J Ultrasonogr 2007; 16: 599-601.

26. Gill S, Hsu TH, Fox RL, et al. Laparoscopic and percutaneous radiofrequency ablation of the kidney: acute and chronic porcine study. Urology 2000; 56: 197-200.

27. Corwin TS, Lindberg G, Traxter O, et al. Laparoscopic radiofrequency thermal ablation of renal tissue with and without hilar occlusion. J Urol 2001; 166: 281-4.

28. Rendon RA, Kachura JR, Sweet JM, et al. The uncertainty of radio frequency treatment of renal carcinoma: finding at immediate and delayed nephrectomy. J Urol 2002; 167: 1587-92.

29. Johnson DB, Duchene DA, Taylor GD, Pearle MS, Cadeddu JA. Contrast-enhanced ultrasound evaluation of radiofrequency ablation of the kidney: reliable imaging of the thermolesion. J Endourol 2005; 19: 248-52. 